Report

\title{
The influence of growth factors on the proliferative potential of normal and primary breast cancer-derived human breast epithelial cells
}

\author{
Stephen P. Ethier ${ }^{1}$, Rochelle M. Summerfelt ${ }^{1}$, Kimberly C. Cundiff ${ }^{1}$ and Bonnie B. Asch ${ }^{2}$ \\ ${ }^{I}$ Department of Radiation Oncology, The University of Michigan Medical Center, Ann Arbor, MI; \\ ${ }^{2}$ Department of Experimental Pathology, Roswell Park Memorial Institute, Buffalo, New York, USA
}

Key words: autocrine factors, cholera toxin, culture methods, epidermal growth factor, growth factor, insulin, mammary epithelial cells, primary cultures

\begin{abstract}
In previous studies, we developed serum-free, bovine pituitary extract (BPE)-free culture conditions for the growth of normal and neoplastic rat mammary epithelial cells. The present studies were aimed at determining if these culture methods could be used to study the influence of specific growth factors on the proliferative potential of normal human mammary epithelial (HME) cells and cells derived from human breast cancer (HBC) specimens. Our results indicate that normal HME cells in primary culture express stringent requirements for insulin (IN), epidermal growth factor (EGF), and cholera toxin (CT). Of these factors, EGF is most important, with essentially no proliferation taking place in the absence of this factor. By contrast, when cells are grown in serum-free primary culture in the presence of a full complement of growth factors and then subcultured, growth in secondary culture is not influenced by the removal of individual growth factors. Growth in secondary culture in the absence of EGF is mediated by autocrine factors secreted by the cells. However, there is no evidence for autocrine activity that mediates growth in the absence of IN in secondary cultures. Primary culture of HBC cells in serum-free, BPE-free medium revealed two patterns of growth factor requirements. One set of HBC cells expressed identical requirements for IN and EGF in primary culture as normal cells. Likewise, these cells grew in secondary culture in the absence of either factor. The second set of tumors expressed independence of IN for growth in primary culture. These cells grew to confluence in primary culture in the absence of IN and could be subcultured in this medium. All tumor cells examined expressed a requirement for EGF for primary culture growth, whereas none of the HBC cells examined expressed a significant CT requirement. In many cases, growth in the absence of CT exceeded that observed in its presence. Thus, our culture system allows analysis of the growth factor requirements of $\mathrm{HME}$ and $\mathrm{HBC}$ cells in primary culture. Our results indicate significant differences between HME and HBC cells in this regard. However, the results of secondary culture experiments indicate that the growth factor milieu from which cells are taken can have a profound effect on the requirements for growth factors in culture.
\end{abstract}

\section{Introduction}

There are now many culture systems based on media that support extended growth of human mammary epithelial cells from normal and neoplastic tissues. The first such medium was devel- 
oped by Stampfer et al. [1]. This medium consists of a combination of serum and growth factor-supplemented fresh medium and conditioned medium obtained from various human cancer cell lines. In this culture medium, normal tissue and human breast cancer derived mammary epithelial cells can be grown in mass culture for one to three passages, and addition of a fibroblast feeder layer growth allows growth at clonal densities [2].

Culture systems that support growth of normal HME cells have now been significantly refined. Growth of these cells over extended periods has been achieved in a growth factor-supplemented serum-free medium containing BPE. For growth over a few passages, the BPE can be replaced by prolactin (M) and prostagladin E-1, allowing growth of HEM cells in a defined medium [3]. Media designed for growth of HBC cells have also been developed. Peterson and van Deurs [4] reported growth of $\mathrm{HBC}$ cells in primary culture in a growth factor-supplemented serum-free medium. More recently, long term growth and serial passage of HBC cells has been reported by Band and Sager [5] using a growth factor-supplemented medium containing both BPE and serum. The emphasis of much of the work performed with the culture systems described above has been on developing conditions suitable for long-term growth of human mammary cells and, whenever possible, establishing cell lines. Consequently, there has been relatively little emphasis placed on the role of individual growth factors on the proliferative potential of the cells in primary and secondary cultures in the absence of the undefined factors present in serum and BPE.

In previous work, we developed culture systems that support extended growth of normal and neoplastic rat mammary epithelial cells under serumfree, BPE-free, conditions [6, 7]. These systems were used to compare directly the proliferative potential and growth factor requirements of normal and neoplastic rat mammary cells [8]. The present studies were designed to determine if the culture methods used to study the proliferative potential of normal and neoplastic rat mammary epithelial cells could be used for studies of HME cell growth and to compare the stowth factor require- ments of normal tissue and breast cancer-derived human mammary cells in primary and secondary culture.

\section{Material and methods}

\section{Preparation of cells for culture}

Normal HME cells were obtained by enzymatic dissociation of normal tissues obtained from reduction mammoplasties or from biopsy specimens. Primary breast cancer specimens were obtained from The University of Michigan Breast Care Center. Enzymatic dissociation of both normal and breast cancer tissue was performed essentially as described previously for rat mammary tissues [9]. Briefly, tissues were minced with scalpels and incubated overnight in type III collagenase (200 units $/ \mathrm{ml}$, Worthington Biochemicals, Freehold, $\mathrm{NJ}$ ) in Medium 199 in a shaking water bath at $37^{\circ} \mathrm{C}$. The cells were collected by centrifugation, washed in fresh Medium 199, and incubated in $0.05 \%$ Pronase (Calbiochem, LaJolla, CA) in Medium 199 at $37^{\circ} \mathrm{C}$. Following the pronase incubation, the cells were pipetted gently to break up large clumps of cells and then washed three times in fresh Medium 199. The number of cells in the suspension was determined by counting isolated nuclei with a Coulter counter as described previously [6]. Epithelial aggregates were either seeded directly into culture or were frozen for future use. Cells were frozen in Medium 199 containing 20\% FBS and 5\% DMSO and freezing was accomplished using a step-freezer.

\section{Tissue culture conditions}

HME and HBC cells were plated at $2 \times 10^{5}$ or $5 \times$ $10^{5}$ cells per $60 \mathrm{~mm}$ culture dish. The plates were coated with a type I collagen film (Vitrogen 100, Collagen Corp, Palo Alto, CA) and rinsed with Hank's balanced salt solution just prior to use. The serum-containing medium consisted of Ham's F-12 (Hazelton Biologicals, Lenexa, KS) supplemented with ethanolamine $(5 \mathrm{mM}), \mathrm{N}$-2-hydroxyethylpip- 
erazine-2-ethane sulfonic acid (HEPES, $10 \mathrm{mM}$ ), IN $(5 \mu \mathrm{g} / \mathrm{ml})$, hydrocortisone $(1 \mu \mathrm{g} / \mathrm{ml})$, EGF $(10 \mathrm{ng} / \mathrm{ml})$, CT $(100 \mathrm{ng} / \mathrm{ml}), \mathrm{M}(5 \mu \mathrm{g} / \mathrm{ml})$, progesterone $(\mathrm{P}, 100 \mathrm{ng} / \mathrm{ml}), 2 \%$ fetal bovine serum (FBS), gentamycin $(5 \mu \mathrm{g} / \mathrm{ml})$, and fungizone $(0.5 \mu \mathrm{g} / \mathrm{ml})$. In the serum-free medium, the FBS was replaced with transferrin $(5 \mu \mathrm{g} / \mathrm{ml})$, bovine serum albumin $(1 \mathrm{mg} / \mathrm{ml})$, sodium selenite $(50 \mathrm{ng} /$ $\mathrm{ml})$, and triiodothyronine $(10 \mathrm{nM})$. Cells were maintained in a humidified atmosphere of $90 \%$ air and $10 \% \mathrm{CO}_{2}$ and were fed three times per week.

\section{Cell growth assays}

To measure the proliferative potential of HME and $\mathrm{HBC}$ cells in various media in primary culture, cells were seeded into $60 \mathrm{~mm}$ dishes and 24 hours later the number of attached cells per dish was determined. The cells were grown for 10 to 12 days with three to four medium changes, and the mean number of cells per dish was determined for each group. Cell counts were performed by isolating nuclei directly from monolayers and counting with a Coulter counter as done previously [9]. For experiments performed in secondary culture, cells were seeded into primary culture and grown for 12 days in the fully growth factor-supplemented serum-free medium. The cells were then sub-cultured and seeded at $10^{5}$ cells per $60 \mathrm{~mm}$ culture dish in growth factorfree medium supplemented with $2 \%$ FBS (to allow cell attachment) overnight. The cells were then switched to different serum-free media in which individual growth factors were deleted and grown for an additional 10 days. To measure the influence of the EGF-receptor-antibody EGFR-425 on HME cell growth, cells were grown in primary culture in fully growth factor-supplemented serumfree medium and then switched in secondary culture to EGF-free medium as described above. The cells were grown in EGF-free medium in the presence or absence of EGFR-425.

Conditioned medium was collected from HME cell cultures by growing the cells in secondary culture in the absence of a single factor. When these secondary cultures became confluent, the cells were re-fed with fresh medium, incubated for an additional 48 hours, and the media collected. To examine mitogenic activity in conditioned medium obtained from HME cell cultures, the normal human mammary epithelial cell line MCF-10 were used as indicator cells [10]. MCF-10 cells were kindly provided by Dr. Herbert Soule of the Michigan Cancer Foundation. MCF-10 cells were seeded at $10^{5}$ cells per $35 \mathrm{~mm}$ culture dish and grown in serum-free medium supplemented with IN, HC, and EGF, or in medium devoid of IN or EGF and containing conditioned medium obtained from HME cells that were grown in secondary culture in the absence of the same factor.

\section{Immunocytochemistry}

To examine cytokeratin expression in HME and $\mathrm{HBC}$ cells, cells were grown in primary culture in the fully growth factor-supplemented serum-free medium on collagen-coated coverslips (Lux, Flow Laboratories, McLean VA). The cells were fixed and stored in cold ethanol and stained by indirect immunofluorescence.

\section{Materials}

The EGFR-425 antibody was kindly provided by Dr. Donald Buchsbaum of The University of Michigan, and the Tab-1 antibody was kindly provided by Dr. Beatrice Langton of Triton Bioscience, Alameda, CA. IN, HC, EGF, M, P, triiodothyronine, sodium selenite, ethanolamine, HEPES, and transferrin were obtained from Sigma Chemical Co. (St. Louis, MO), CT was from Schwartz-Mann (Spring Valley, NY), Vitrogen was from the Collagen Corporation (Palo Alto, CA), and Ham's F-12 was from Hazelton Biologicals (Lenexa, KS). Lux coverslips were obtained from Flow Laboratories (McLean, VA). 


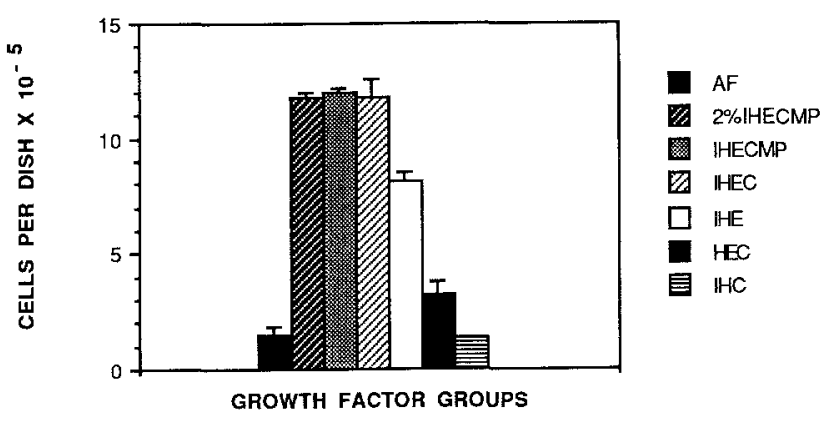

Fig. 1. Primary culture of HME cells obtained from a reduction mammoplasty in $2 \%$ serum-containing or serum-free medium in the presence of a full complement of growth factors or in the absence of individual factors. The cells were grown for 12 days and the number of cells per dish was determined by counting isolated nuclei with a Coulter counter. The attached fraction (AF) indicates the number of attached cells per dish 24 hours after plating. The legends for the individual bars indicate the growth factors present in each medium; $\mathrm{I}=$ insulin, $\mathrm{H}=$ hydrocortisone, $\mathrm{E}=$ epidermal growth factor, $\mathrm{C}=$ cholera toxin. In all figures, error bars indicate the range.

\section{Results}

Growth factor requirements of HME cells in primary culture

To examine the influence of individual growth factors on the proliferative potential of normal HME cells in primary culture, epithelial aggregates were obtained by enzymatic dissociation of one reduction mammoplasty specimen and two biopsy specimens that consisted of histologically normal mammary tissue. The cells were plated at low density on collagen-coated $60 \mathrm{~mm}$ culture dishes and allowed to attach overnight in HEPES-buffered Ham's F-12 medium supplemented with ethanolamine, hydrocortisone, and 2\% FBS (plating medium). The next day, attached cells were washed and switched to serum-free medium supplemented with various growth factor combinations. Also at this time, the number of attached cells in triplicate plates was determined. The cells were grown for 12 days with four medium changes, and the number of cells per dish was determined by counting isolated nuclei. The results of a primary culture deletion experiment performed with cells from the reduction mammoplasty specimen are shown in Fig. 1.

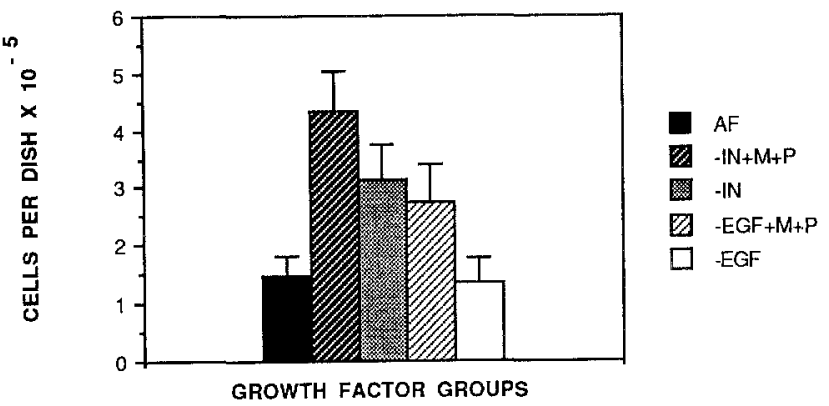

Fig. 2. Influence of progesterone (P) and prolactin (M) on HME cell growth in primary culture in the absence of either insulin (IN) or EGF. The experiment was performed as described in Fig. 1. - IN or - EGF indicate growth in the absence of the growth factor.

Over the 12 day culture period, growth in the fully growth factor-supplemented serum-free medium was equivalent to that obtained in a similar medium that also contained $2 \%$ FBS. Also, deletion of $\mathrm{M}$ and $\mathbf{P}$ from the medium did not result in any decrease in primary culture growth. By contrast, deletion of CT resulted in an approximately 50\% reduction in cell growth, and deletion of either IN or EGF dramatically reduced the proliferative potential of the cells. Thus, for these HME cells, as for rat mammary epithelial cells [7] and other cultured epithelial cell types [11-14], there is a synergistic interaction between IN and EGF in stimulating cell growth.

Although addition of $\mathrm{M}$ and $\mathrm{P}$ to the complete culture medium did not result in enhanced cell proliferation, these hormones did enhance growth when tested in the absence of either IN or EGF. The data in Fig. 2 show that growth in the absence of IN or EGF was greater in the presence of $M$ and $P$ than in their absence. Thus, under sub-optimal culture conditions, mammogenic hormones do have detectable effects on HME proliferation.

Primary culture deletion experiments were also performed with two biopsy samples that were shown to be histologically normal, and the results of these experiments are given in Figs $3 \mathrm{~A}$ and $\mathrm{B}$. These results confirm the results of experiments with reduction mammoplasty-derived cells and indicate that both IN and EGF are required for HME cell growth. However, the cells from these two 

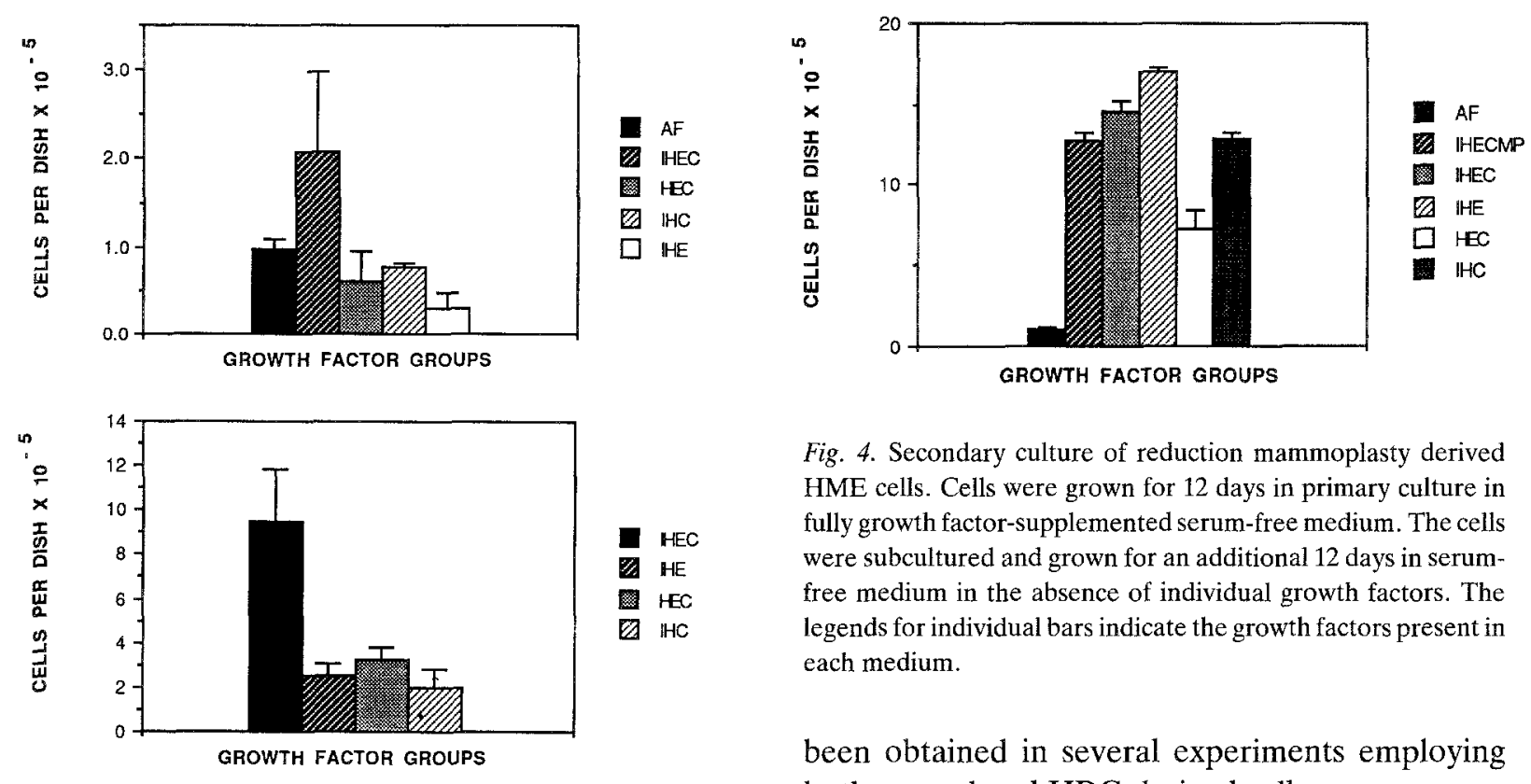

Fig. 3. Primary culture growth of HME cells obtained from two different biopsy specimens that were shown histologically to consist of normal mammary epithelium. Cells were grown in serum-free medium in the presence of IN, HC, EGF, and CT (IHEC) or in the absence of one factor. Cells were grown for 12 days and then counted as described above.

samples exhibited much greater dependence on CT than did the reduction mammoplasty cells. This finding is consistent with results reported previously that indicate sample to sample variability with regard to CT requirements for in vitro growth [15].

The above results indicate that HME cells respond dramatically to the presence or absence of individual growth factors in primary culture. To determine if the cells respond similarly in secondary cultures, HME cells were plated as described above and grown for 12 days in serum-free medium in the presence of a full complement of factors. The cells were then sub-cultured and grown for seven days in medium devoid of specific factors. The data in Fig. 4 show that the cells exhibited little or no sensitivity to growth factor withdrawal in the first passage. The cells grew as well in the absence as in the presence of CT or EGF. Growth in the absence of IN was reduced in comparison to the other groups, but the fold-increase in cell number was much greater in secondary culture than in primary culture in IN-minus medium. Similar results have

Fig. 4. Secondary culture of reduction mammoplasty derived HME cells. Cells were grown for 12 days in primary culture in fully growth factor-supplemented serum-free medium. The cells were subcultured and grown for an additional 12 days in serumfree medium in the absence of individual growth factors. The legends for individual bars indicate the growth factors present in each medium.

been obtained in several experiments employing both normal and HBC-derived cells.

To determine if autocrine growth factor synthesis could account for the lack of growth factor requirements in secondary culture, experiments were performed using the newly developed human mammary epithelial cell line MCF-10 as indicator cells. These cells can be serially propagated in our serum-free medium and have an absolute requirement for both IN and EGF. For these experiments, HME cells were grown for 12 days in primary culture in fully growth factor-supplemented medium, subcultured, and grown for an additional seven to ten days in medium devoid of either EGF or IN. When these secondary cultures became nearly confluent (after three medium changes), the cells were re-fed with fresh medium and incubated for an additional 48 hours, and these conditioned media were collected. The conditioned media obtained in this way were tested for their ability to support growth of MCF-10 cells in the absence of either IN or EGF (Fig. 5). The results from this experiment show that MCF-10 cells are exquisitely sensitive to IN and EGF, and that conditioned media obtained from secondary cultures contain potent EGF-like activity but no detectable IN-like bioactivity. To determine if the activity present in the conditioned medium from minus-EGF secondary cultures was responsible for the growth of the cells under these conditions, experiments were performed using the 


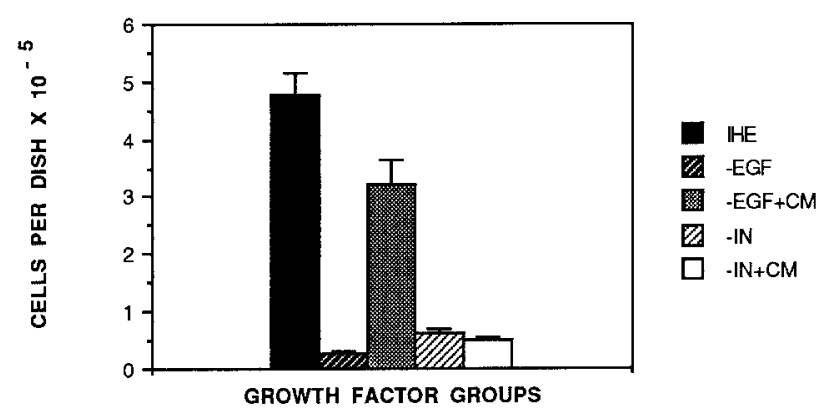

Fig. 5. The influence of conditioned medium, obtained from secondary cultures of HME cells grown in the absence of either IN or EGF, on growth of MCF-10 cells. Conditioned medium was obtained from secondary HME cells as described in Methods. MCF-10 cells were grown in serum-free medium in the presence of IN, HC, and EGF (IHE) or in the absence of either IN or EGF. Cells grown in the absence of either IN or EGF were tested for their response to HME-derived conditioned medium obtained from secondary cultures grown in the absence of the same factor.

neutralizing EGF-receptor antibody EGFR-425 [16]. HME cells were grown in primary culture in fully supplemented medium, and then grown in secondary culture in the absence of EGF and in the presence or absence of EGFR-425. The results indicate that growth in the secondary cultures in the absence of EGF is abolished by the EGF-receptor antibody (Fig. 6).

It has been reported that culture of HME cells results in the synthesis of TGF-alpha by the cells [17]. Therefore, we attempted to measure TGFalpha by radioimmunoassay in conditioned medium obtained from secondary cultures of HME cells. Thus far we have not detected TGF-alpha immunoreactive material in conditioned medium that contains EGF-like bioactivity. Furthermore, growth of HME cells in secondary culture in the absence of EGF was not inhibited by incubation with the TGF-alpha neutralizing antibody (Table 1 ), even at concentrations as high as $100 \mu \mathrm{g} / \mathrm{ml}$ (data not shown). Thus, the identity of the EGFlike activity secreted by HME cells remains to be determined.

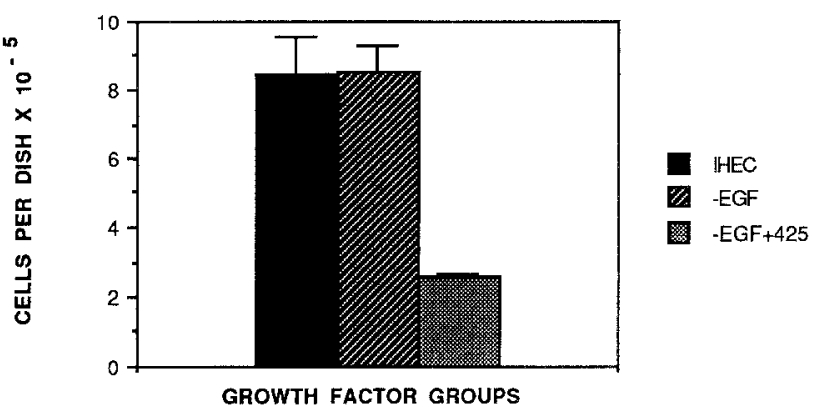

Fig. 6. Influence of the EGF-receptor antibody EGFR-425 on growth of secondary HME cell cultures in the absence of EGF. HME cells were grown in primary culture in fully growth factorsupplemented serum-free medium, subcultured, and grown in secondary culture in the absence of EGF and in the presence or absence of $1 \mu \mathrm{g} / \mathrm{ml}$ EGFR-425.

Growth factor requirements of $\mathrm{HBC}$-derived cells in primary culture

Since primary culture deletion experiments are informative with regard to the growth factor requirements of HME cells, similar experiments were performed with cells derived by enzymatic dissociation of HBC specimens. Thus far we have obtained 15 HBC samples and have successfully cultured cells from 12 of them. Of these 12 , primary culture deletion experiments have been performed on 8 samples and interpretable results have been obtained from 7 of the 8 experiments. Thus far, two patterns of growth factor requirements have been detected. The data obtained from the cells designated SUM-13 are representative of one pattern, in which the requirements for IN and EGF are indistinguishable from those of normal cells but which show better growth in the absence than in the presence of CT (Fig. 7). Also presented in Fig. 7 are the results of secondary culture deletion experiments that show the same loss of growth factor sensitivity for HBC cells in the first passage as for normal tissue-derived HME cells.

The data obtained from SUM-18 are representative of the second pattern of primary culture growth observed thus far. These cells expressed significant growth potential in primary culture in the absence of IN as well as in the absence of CT (Fig. 8). Cells derived from these tumors grew to confluence in primary culture in the absence of IN and were 

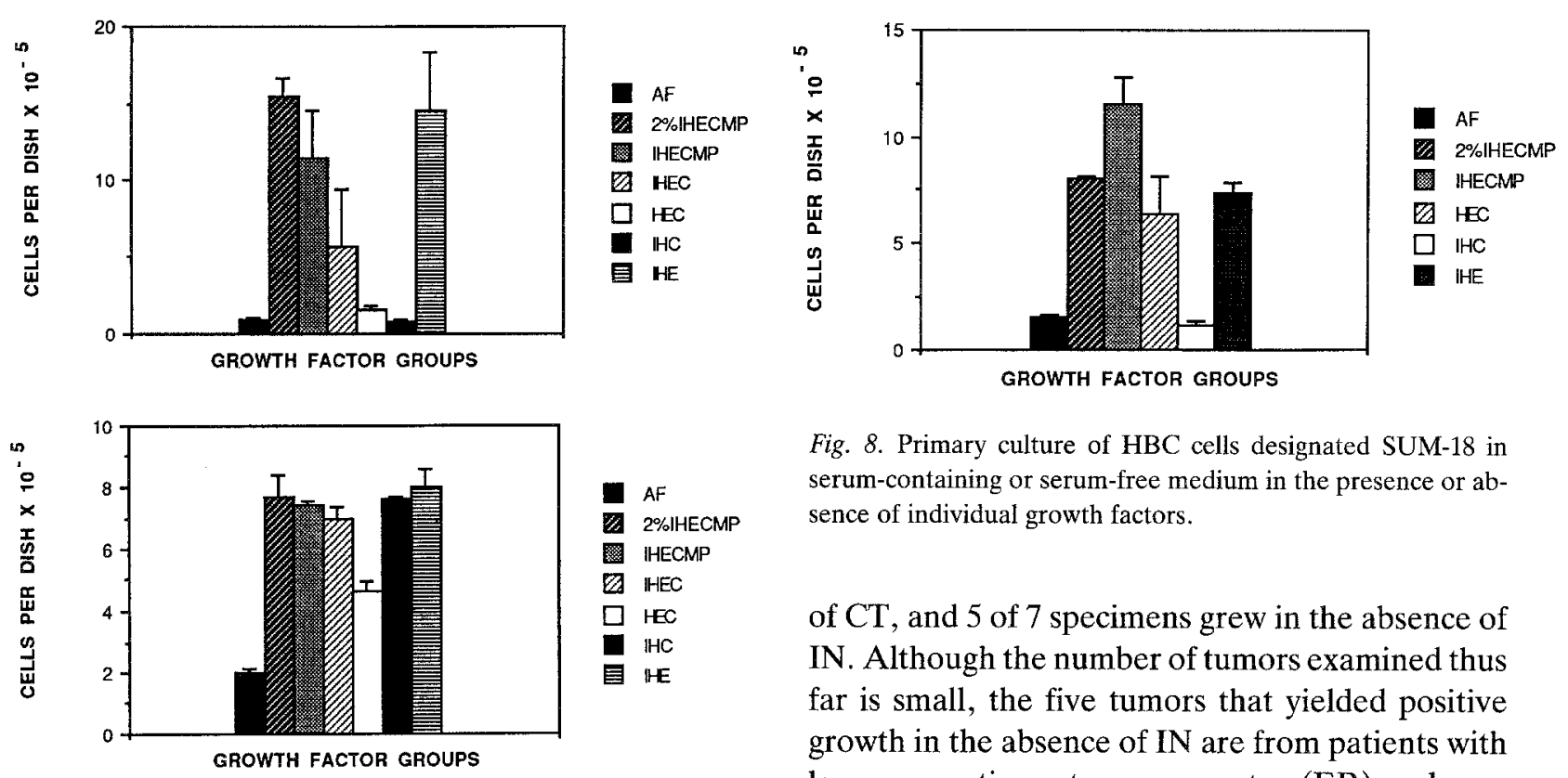

Fig. 8. Primary culture of HBC cells designated SUM-18 in serum-containing or serum-free medium in the presence or absence of individual growth factors.

of CT, and 5 of 7 specimens grew in the absence of IN. Although the number of tumors examined thus far is small, the five tumors that yielded positive growth in the absence of IN are from patients with low or negative estrogen receptor (ER) and progesterone receptor (PR) levels, and were taken

Fig. 7. Primary culture (A) and secondary culture (B) of HBC cells designated SUM-13 in serum-containing and serum-free medium in the presence or absence of individual growth factors. The experiments were performed as described in Figs 1 and 4.

subcultured. This level of growth in IN-free medium is many fold greater than anything that we have observed with normal tissue-derived cells.

The results obtained with the seven primary tumors analysed to date are summarized in Table 1. It is noteworthy that 5 of 5 specimens tested expressed excellent growth potential in the absence from patients that had locally advanced and/or metastatic disease.

\section{Cytokeratin expression in proliferating HME and $H B C$-derived cultures}

The culture conditions used in these experiments were originally developed for growth of normal and neoplastic rat mammary epithelial cells and to minimize growth of stromal cells [9]. The HME and

Table 1. Characteristics of human breast cancers for which primary culture deletion data are available

\begin{tabular}{|c|c|c|c|c|c|c|}
\hline Tumor designation & Histo/path $\mathrm{Dx}^{\mathrm{a}}$ & Node status & $\mathrm{ER}^{\mathrm{b}}$ & $\mathrm{PR}^{\mathrm{b}}$ & Growth $-\mathrm{IN}^{\mathrm{c}}$ & Growth $-\mathrm{CT}^{\mathrm{c}}$ \\
\hline SUM-12 & IC, LCIS medullary CA & neg & 12 & neg & - & + \\
\hline SUM-13 & Invasive duct. $\mathrm{CA}$ & neg & 875 & 658 & - & + \\
\hline SUM-4 & Invasive duct. CA & neg & neg & neg & + & + \\
\hline SUM-7 & Invasive duct. $\mathrm{CA}$ & $11 / 13^{\mathrm{d}}$ & 50 & neg & + & $\mathrm{NT}^{\mathrm{e}}$ \\
\hline SUM-16 & Invasive duct. CA & $15 / 15^{\mathrm{d}}$ & neg & neg & + & $\mathrm{NT}^{\mathrm{e}}$ \\
\hline SUM-18 & Invasive duct. CA & neg & 12 & 147 & + & + \\
\hline SUM-23 & Invasive duct. CA & neg & 12 & neg & + & + \\
\hline
\end{tabular}

\footnotetext{
a IC, intraductal carcinoma; LCIS, lobular carcinoma in situ; Invasive duct. CA, invasive ductal carcinoma.

${ }^{\mathrm{b}}$ Estrogen and progesterone receptor levels in fmols per mg protein.

${ }^{c}$ Ability of the cells to grow to confluence in primary culture in the absence of either IN or CT.

${ }^{\mathrm{d}}$ Number of nodes positive for breast cancer/number of nodes examined.

e NT, not tested.
} 
HBC cells that grew under these culture conditions exhibited a typical epithelial cell morphology. Furthermore, the majority of HME and HBC cells reacted positively with a broad spectrum antikeratin antiserum, and less than $5 \%$ of the cells expressed vimentin detectable by immunofluorescence.

In summary, the results indicate that our serumfree primary culture system can be used to analyze the growth factor requirements of normal and primary breast cancer-derived human mammary epithelial cells. Our results also indicate that the requirements of HME cells for growth factors are dramatically influenced by the culture environment, such that first passage cells express markedly different growth factor requirements than do primary cells. Furthermore, cells derived from some primary HBC express altered requirements for IN and $\mathrm{CT}$ for growth in primary culture.

\section{Discussion}

The results of these experiments indicate that the growth factor requirements of normal and human breast cancer-derived mammary epithelial cells can be compared in serum-free, BPE-free primary culture. The culture medium used for these experiments was originally developed for our studies of growth of normal and neoplastic rat mamary epithelial cells. Although there are many similarities between our medium and those used by other groups for growth of human mammary epithelial cells $[3,5]$, there are significant differences. First, we use Ham's F-12 as the basal medium, which in our hands is superior to other formulations, including 50: $50 \mathrm{mix}$ of F-12 and DMEM. Second, primary and secondary culture growth factor experiments are performed in the absence of either serum or BPE. Indeed, BPE does not improve growth of HME or HBC cells in primary culture in our serum-free medium. Thus, our culture medium most closely resembles the medium developed by Peterson and van Deurs [4]. Our serum-free medium does contain BSA which is not completely defined. The BSA is greater than $99 \%$ pure; however, previous studies have shown that fatty acids associated with the BSA are important to the growth potential of the cells [6]. Thus, BSA in the growth medium is important for maintenance of the proper osmotic pressure and as a source of fatty acids. The latter function is physiological, in that fatty acids are toxic in free form and are presented to cells in vivo associated with BSA. There is no evidence that BSA is a source of undefined growth factors, and neither rat nor human mammary epithelial cells express any growth in BSA-containing medium that is not supplemented with exogenous growth factors.

In primary culture, HME cells exhibit sensitivity to individual growth factors. These cells, like many other cultured epithelial cell types, grow rapidly in the presence of IN, HC, EGF, and a cAMP agonist such as CT. Deletion of IN, EGF, or CT results in dramatic loss of growth potential. When the same cells are first grown in the presence of a full complement of factors and then tested for responsiveness to deletion of individual factors, dramatically different results are obtained. HME cells (and HBC cells) grow as well in the absence as in the presence of either EGF or CT in secondary culture. In addition, growth in secondary culture in the absence of IN is much greater than that seen in minus-IN primary cultures. The ability of HME cells to grow in secondary culture in the absence of EGF is mediated by autocrine factors secreted into the medium. By contrast, there is no IN-like activity in conditioned medium obtained from cultures grown in the absence of IN. The results obtained in these experiments are consistent with those obtained in other laboratories that indicate up-regulation of growth factors, growth factor receptors, and protooncogenes upon initiation of primary culture of HME cells [17-19].

Primary culture experiments performed with cells obtained from primary breast cancers revealed two patterns of growth factor response. First, all tumors examined thus far grew well in primary culture in the absence of CT. In many cases growth in the absence of CT is greater than that observed in its presence. Second, 5 of 7 tumors examined have expressed good growth in primary culture in the absence of IN. These cells come to confluence in primary culture in minus-IN medium 
and can be serially propagated in the absence of this factor. These results represent clear differences between the response of HME and HBC cells to growth factors in primary culture. However, the results must be interpreted in light of the data obtained in the secondary culture experiments performed with $\mathrm{HME}$ and $\mathrm{HBC}$ cells. The ability to grow in primary culture in the absence of individual growth factors may reflect genetic changes important to the neoplastic potential of the $\mathrm{HBC}$ cells. Alternatively, this phenotype may be a reflection of the environment from which the $\mathrm{HBC}$ cells were obtained. That is, the milieu of the primary tumor may be similar to that of the primary culture environment where growth factors are present. Thus, placing cells in primary culture from such an environment may be akin to starting secondary cultures of normal cells following primary culture in the presence of growth factors. Distinguishing between these two possibilities will provide a significant insight into the factors that drive proliferation of HBC cells in vivo.

Immunocytochemical analysis of cells grown from normal and neoplastic tissues indicates that they are epithelial cells. However, it is much more difficult to state unequivocally that cells grown from breast cancer specimens are indeed neoplastic cells. In an attempt to minimize the presence of normal mammary elements in HBC samples, we have worked with samples taken from patients entering a clinical protocol for locally advanced disease. These patients do not receive mastectomies but rather receive open biopsies for staging purposes. Thus, our laboratory receives tumor pieces from the central areas of large lesions that are sampled on the basis of their gross neoplastic appearance. Since these biopsies are for staging purposes, they are given careful histopathologic evaluation. Therefore, the source of the cells used in these experiments and their altered growth factor requirements in serum-free culture suggest that they are indeed human breast cancer cells. However, further work will be required to demonstrate this unequivocally.

\section{References}

1. Stampfer MR, Hallowes RC, Hackett AJ: Growth of normal human mammary epithelial cells in culture. In Vitro 16: 415-425, 1980

2. Smith HS, Lan S, Ceriani R, Hackett AJ, Stampfer MR: Clonal proliferation of cultured nonmalignant and malignant human breast epithelia. Cancer Res 41: 4637-4643, 1981

3. Hammond SL, Ham RG, Stampfer MR: Serum-free growth of human mammary epithelial cells: rapid clonal growth in defined medium and extended serial passage with pituitary extract. Proc Natl Acad Sci USA 81: 5435-5439, 1984

4. Petersen OW, van Deurs B: Preservation of defined phenotypic traits in short-term cultured human breast carcinoma derived epithelial cells. Cancer Res 47: 858-866, 1987

5. Band V, Sager R: Distinctive traits of normal and tumor derived human mammary epithelial cells expressed in a medium that supports long-term growth of both cell types. Proc Natl Acad Sci USA 86: 1249-1253, 1989

6. Ethier SP: Serum-free culture conditions for the growth of normal rat mammary epithelial cells in primary culture. In Vitro: Cell Devel Biol 22: 485-490, 1986

7. Ethier SP, Kudla A, Cundiff KC: The influence of hormone and growth factor interactions on the proliferative potential of normal rat mammary epithelial cells in vitro. J Cell Physiol 132: 161-167, 1987

8. Ethier SP, Cundiff $\mathrm{KC}$ : Importance of extended growth potential and growth factor independence on in vivo neoplastic potential of primary rat mammary carcinoma cells. Cancer Res 47: 5316-5322, 1987

9. Ethier SP: Primary culture and serial passage of normal and carcinogen-treated rat mammary epithelial cells in vitro. $\mathbf{J}$ Natl Cancer Inst 74: 1307-1318, 1985

10. Soule HD, Malony TM, Wolman SR, Peterson WD, Brenz R, McGrath CM, Russo J, Pauly RJ, Jones RF, Brooks SC: Isolation and characterization of a spontaneously immortalized human breast epithelial cell line, MCF-10. Cancer Res. 50: 6075-6086, 1990

11. Babcock MS, Marino MR, Gunning WT, Stoner GD: Clonal growth and serial propagation of rat esophageal epithelial cells. In Vitro 19: 403-415, 1983

12. Bertolero F, Kaighn ME, Gonda MA, Safiotti U: Mouse epidermal keratinocytes: Clonal proliferation and response to hormones and growth factors in serum-free medium. Exp Cell Res 155: 64-80, 1984

13. Lechner JF, Jaugen A, McClendon IA, Pettis WE: Clonal growth of normal adult human bronchial epithelial cells in a serum-free medium. In Vitro 18: 633-642, 1982

14. Wille JJ, Pittelkow MR, Shipley CD, Scott RE: Integrated control of growth and differentiation of normal human prokeratinocytes cultured in serum-free media: Clonal analysis, growth kinetics, and cell cycle studies. J Cell Physiol 121: 34-44, 1984 
15. Stampfer MR: Cholera toxin stimulation of human mammary epithelial cells in culture. In Vitro 18: 531-537, 1982

16. Rodeck U, Herlyn M, Herlyn D, Molthoff C, Atkinson B, Varello M, Steplewski Z, Koprowski H: Tumor growth modulation by a monoclonal antibody to the epidermal growth factor receptor: Immunologically mediated and effector cell-independent effects. Cancer Res 47: 3692-3696, 1987

17. Zajchowski D, Band V, Pauzie N, Tager A, Stampfer M, Sager R: Expression of growth factors and oncogenes in normal and tumor-derived human mammary epithelial cells. Cancer Res 48: 7041-7047, 1988
18. Valverius EM, Bates SE, Stampfer MR, Clark R, McCormick F, Salomon DS, Lippman ME, Dickson RB: Transforming growth factor-alpha production and epidermal growth factor receptor expression in normal and oncogene transformed human mammary epithelial cells. Mol Endocrinol 3: 203-214, 1989

19. Bates SE, Valverius EM, Ennis BW, Bronzert DA, Sheridan JP, Stampfer MR, Mendelson J, Lippman ME, Dickson RB: Expression of the transforming growth factor alpha/epidermal growth factor receptor pathway in normal human breast epithelial cells. Endocrinology 126: 596-607, 1990 